\title{
Macro-mesoporous silicas complex and the carbon replica
}

\author{
Junming Sun ${ }^{\mathrm{a},{ }^{*}}$, Ding Ma ${ }^{\mathrm{a}}$, He Zhang ${ }^{\mathrm{a}}$, Xinhe Bao ${ }^{\mathrm{a}, *}$, Gisela Weinberg ${ }^{\mathrm{b}}$, Dangsheng Su ${ }^{\mathrm{b}}$ \\ ${ }^{a}$ State Key Laboratory of Catalysis, Dalian Institute of Chemical Physics, Chinese Academy of Sciences, \\ Dalian 116023, PR China \\ ${ }^{\mathrm{b}}$ Department of Inorganic Chemistry, Fritz-Haber Institute of the Max Planck Society, Berlin D-14195, \\ Germany \\ * Corresponding authors. E-mail addresses: dlsjm@dicp.ac.cn (J. Sun), xhbao@dicp.ac.cn (X. Bao).
}

\begin{abstract}
Novel macroporous silicas with ordered mesoporous wall structures ( $15 \mathrm{~nm}$ in pore size) have been synthesized by finely balancing the emulsification of the oil phase with the self-assembly of the amphiphilic block copolymers. The nanocasting method was used to produce hierarchically ordered macro-mesoporous carbon materials. These porous materials have potential applications in catalysis, sorption, separation, etc.
\end{abstract}

Keywords: Hierarchy; Macro-mesoporous; Silica; Carbon; Emulsion; Hydrophobic-hydrophilic balance

\section{Introduction}

Since the first report of self-assembled mesoporous silicas [1], great efforts have been made to construct inorganic hierarchical structures [2], especially materials with multimodal porosity [3], which is of great importance in the fields of controlled drug-delivery, catalysis, adsorption, and other energy-related applications.

Various approaches have been invented for the fabrication of multimodal porous materials, which are well summarized in recent review papers [4,5]. Emulsion templating has been considered one of the most promising routes to produce macroporous materials with tunable pore sizes from $50 \mathrm{~nm}$ to a few micrometers [6]. The introduction of macroporosity into mesoporous materials by emulsion templating, however, was found to be far more difficult than expected [7]. For example, for the amphiphilic block copolymers involved emulsion systems [8], strong interactions between oil phase and amphiphilic block copolymers, especially when large amounts of oil (e.g., TMB) was used, often leads to the destruction of mesoscale ordering, forming a lamellar [9] or mesocellular foam (MCF) structures eventually [10]. On the other hand, systems with weaker oil-surfactant interactions are not easily emulsified. As a result, very large hollow spheres (a few micrometers in diameter) with mesoporous walls were obtained, in which high intrusion energy (high stir speed) was often involved [11]. In other works, vesicle structures [12], giant surfactant superstructures [13,14], even bubbles etc. have been used to assist in the construction of macro-mesoporous materials. Su et al. [14] have synthesized a set of hierarchically macro-mesoporous metal oxide in a single surfactant system, in which the templating of giant surfactant superstructures (from unreacted excess surfactant) is responsible for the formation of channel-like macropore systems. However, this approach does not work to prepare silica systems with similar porous structures [15]. To date, only hard template routes, such as colloidal crystals, have been successfully used in the construction of macro-mesoporous materials [4]. It remains a challenge to find a facile and efficient route to the fabrication of hierarchically ordered macro-mesostructures. Our previous studies show that different alkanes have different interactions with P123 amphiphilic block copolymer. By tuning the initial reaction temperatures and compositions of the synthetic mixture, control of inorganic-organic micelle arrangements may be achieved. As a result, mesoporous silicas with tunable pore size and morph-architectures were successfully obtained [16]. Herein, we report the synthesis of a new type of hierarchical macroporous silicas with ordered mesoporous wall structures (pore size 
around $15 \mathrm{~nm})$. At the same time, carbon materials with the same hierarchical macromesostructures are also successfully replicated by a simple nanocasting route.

\section{Experimental}

2.1. Materials synthesis

P123 ( $\mathrm{EO}_{20} \mathrm{PO}_{70} \mathrm{EO}_{20}$ ) (Aldrich Chemical Company), tetraethyl orthosilicate (TEOS) (Acros, 98\%), Hydrochloric acid (36-40 wt.\%) and Hexane (AR) (Shenyang Federal Regeant Co., Ltd.). $\mathrm{NH}_{4} \mathrm{~F}$ (AR) (Tianjin Kermel Chemical Reagent Co., Ltd.). All the regents were used as received without further purification.

The typical synthesis of macro-mesoporous silicas was performed as follows: pre-mixture of hexane and TEOS was added into the $\mathrm{EO}_{20} \mathrm{PO}_{70} \mathrm{EO}_{20}(\mathrm{P} 123)-\mathrm{H}_{2} \mathrm{O}-\mathrm{HCl}-\mathrm{NH}_{4} \mathrm{~F}$ solutions (final P123: $\mathrm{HCl}: \mathrm{NH}_{4} \mathrm{~F}: \mathrm{H}_{2} \mathrm{O}$ :hexane:TEOS molar ratios $=1: 261: 1.8: 11278: 235: 54$ ) under a moderate mechanical stir. The synthetic mixture was further stirred at $293 \mathrm{~K}$ for $20 \mathrm{~h}$, transferred into an autoclave, and kept at $373 \mathrm{~K}$ for $48 \mathrm{~h}$. The products were collected by filtration, dried in air, and calcined at $813 \mathrm{~K}$ for $5 \mathrm{~h}$ to remove the templates.

The macro-mesoporous carbon materials were prepared according to the publishing procedure with minor revisions [17]. Briefly, 5 g sucrose was dissolved into $20 \mathrm{ml}$ distilled water, which is followed by addition of ca. $0.60 \mathrm{~g} \mathrm{H}_{2} \mathrm{SO}_{4}$. Calcined macro-mesoporous silicas (1.0 g) were mixed with $10 \mathrm{ml}$ of the above prepared solution. The mixture was placed in an oven for $6 \mathrm{~h}$ at $373 \mathrm{~K}$, and then heated to $423 \mathrm{~K}$ where the mixture was further treated for another $6 \mathrm{~h}$. Upon cooling, the obtained macro-mesoporous silicas with partially polymerized and carbonized sucrose were mixed again with $8 \mathrm{ml}$ of the above mentioned sucrose/ water $/ \mathrm{H}_{2} \mathrm{SO}_{4}$ solutions, which was treated again at $373 \mathrm{~K}$ and $423 \mathrm{~K}$. Carbonization was carried out at $1073 \mathrm{~K}$ under flowing helium (30 ml/min) for ca. $2 \mathrm{~h}$. The obtained carbon- silica composite was treated with $5 \%$ hydrofluoric acid under ultrasonic agitation to get rid of the silica template. After two cycles of hydrofluoric acid treatment, the macro- mesoporous carbon materials were thus obtained by filtration, washing and drying.

\subsection{Characterization}

SEM (scanning electron microscope) was done on a Hitachi S4800 Field-emisson scanning electron microscope. The TEM (transition electron microscope) image was obtained with a Philips CM 200 transmission electron microscope equipped with a CCD camera. XRD patterns were collected on a Rigaku D/MAX 2400 diffractometer equipped with a $\mathrm{Cu} \mathrm{K}_{\alpha} \mathrm{X}$-ray source operating at $40 \mathrm{kV}$ and $50 \mathrm{~mA}$. The $\mathrm{N}_{2}$ adsorption-desorption isotherms were recorded on a micromeritics ASAP 2000 instrument.

\section{Results and discussions}

Fig. 1a shows the HRSEM image (more SEM images in Fig. S1) of the obtained macromesoporous silicas. It is clear that they are mainly fused hollow spherical particles. Significantly, the walls of those hollow particles consist of circular mesochannels which curl around side by side to construct the outer spherical shell (white arrow marked). Fig. 1b gives the STEM image of the same area. Surprisingly, the observation with low energy electron beam (accelerating voltage is $30 \mathrm{kV}$ in this case) gives clearlyresolved image of the large particles, revealing the macroporous nature of the hierarchical macro-mesoporous structures. This phenomenon suggests that the materials thus obtained have a hierarchically porous structure of hollow spherical shells which are further composed of curling mesochannels on the surface, making a multimodal pore system in the final product. Such a structure is also consistent with HRSEM observations (Fig. 1a). In addition, the diameter of the inner voids is estimated to be around 200$250 \mathrm{~nm}$. TEM image in Fig. 1d (more TEM images available in Fig. S2) gives better insight into the inner structures of the obtained materials. At the same time, the mesopore size estimated from TEM is about $15 \mathrm{~nm}$. To our best knowledge, it is the first report on the construction of this type of hierarchically ordered macro-mesoporous materials by employing a multi-scale selfassembly process. 

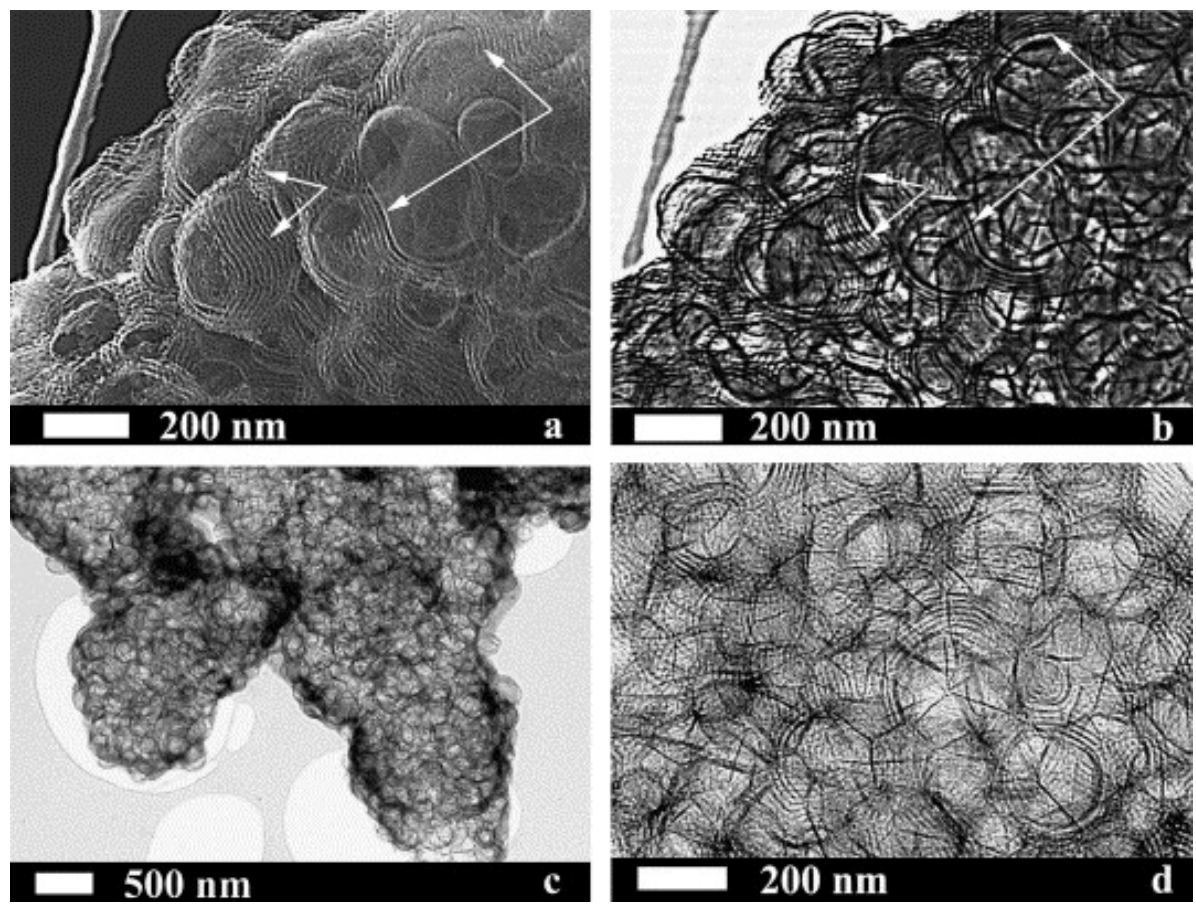

Fig. 1.HRSEM (a), STEM (b) and TEM (c and d) images of the hierarchically structured macro-mesoporous silicas.

Nitrogen sorption isotherms (type IV) of the obtained hierarchically macro-mesoporous silicas (Fig. 2a) give two nitrogen condensation steps at relative pressure above 0.80 , indicating that the obtained materials possess two set of pore channels. The first step at relative pressure of ca. 0.85 could be attributed to the nitrogen capillary condensation in the mesoporous silicas nanotubes [16]. Another nitrogen adsorption step occurred at higher relative pressure (more than 0.9) might be attributed to the nitrogen adsorption in the macroporous systems, which could be further confirmed by the EM observations. The pore size distribution (calculated from the adsorption branch of the isotherms by BJH (Barrett-Joyner-Halenda) model) shows a maximum at $15.2 \mathrm{~nm}$ (inset in Fig. 2a), which agrees well with that detected by (calculated from the adsorption branch
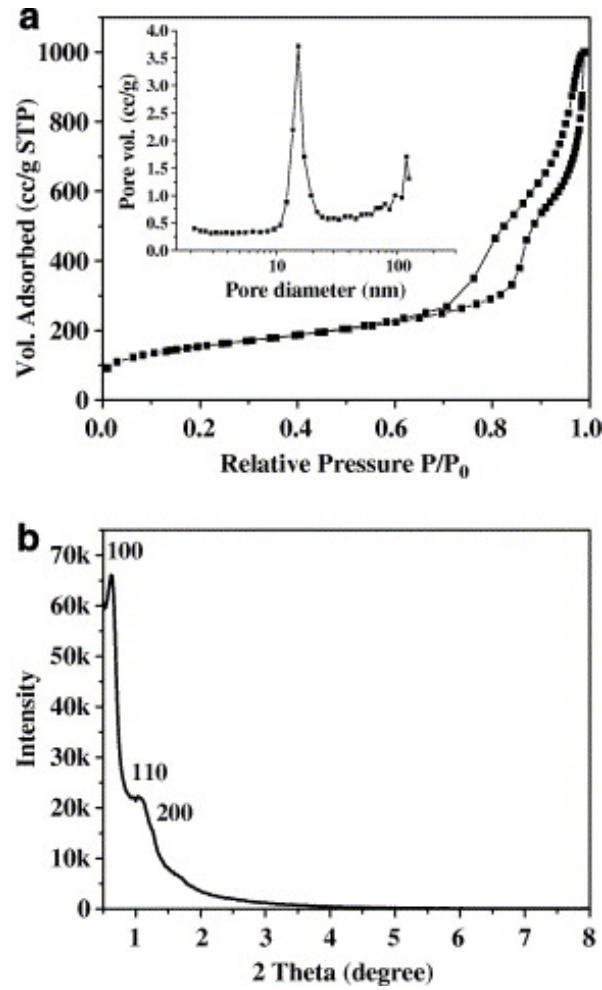

Fig. 2.Nitrogen sorption isotherms (a) and SAXD pattern (b) of the hierarchical macro-mesoporous silicas. Inset in Fig. 2a is pore size distribution calculated from the adsorption branch by BJH method. 
of the isotherms by BJH (Barrett-Joyner-Halenda) model) shows a maximum at $15.2 \mathrm{~nm}$ (inset in Fig. 2a), which agrees well with that detected by TEM. At the same time, the BET surface area and pore volume of the macro-mesoporous silicas are $546 \mathrm{~m}^{2} / \mathrm{g}$ and $1.56 \mathrm{~cm}^{3} / \mathrm{g}$, respectively. SAXD (small-angle X-ray diffraction) pattern gives one intensive diffraction peak at $0.62^{\circ}$ (Fig. 2b), with a broad hump at ca. $1.04^{\circ}$ and a shoulder at ca. $1.28^{\circ}$, which could be assinged to (100), (110) and (200) diffractions of the 2-D hexagonal mesostructure (P6mm symmetry and unit cell parameter, $\mathrm{a}=16.4 \mathrm{~nm}$ ), respectively. The observation is consistent with the TEM and nitrogen sorption results.

Previous studies indicate that the match between amphiphilic triblock copolymers and immiscible solvents (i.e., the balance of their hydrophobic and hydrophilic properties [18]) plays a key role in controlling the assembly of silicate- amphiphilic block copolymers complex in the water phase [16]. Normally, strong interactions often lead to disordered structures (e.g., MCFs), especially in the presence of a large amount of cosolvents [10]. In this case, a proper ratio of oil/water/surfactant/silicate and initial reaction temperature are essential to the formation of hierarchical emulsion system, that is, surfactant protected oil droplets (in this case, $250 \mathrm{~nm}$ in size) are wrapped with circular hexagonal- ordered silicate-surfactant complex (Scheme 1). The circle-ordered arrangement of the surfactant monolayer is believed to be responsible for the circular patterning of hexagonal-ordered silicate-doped micelles. Upon hydrothemal treatment, oil droplets supported hexagonal mesostructured silicas were formed. After drying at $373 \mathrm{~K}$ and calcination at $813 \mathrm{~K}$, both the oil phase and the surfactants occluded inside the mesoporous channels were removed, and novel hierarchically ordered macro- mesoporous silicas are thus obtained. It is noteworthy that a slight deviation from the currently employed reaction temperatures and compositions (i.e., TEOS/P123 $=40$ or 60 ) would not lead to the formation of the present macro-mesoporous materials, but instead the formation of SBA-15 or MCFs (not shown here), indicating that the formation of this ordered macro-mesoporous silicas only occurs with a strict control of surfactant/silicate-doped surfactant properties.

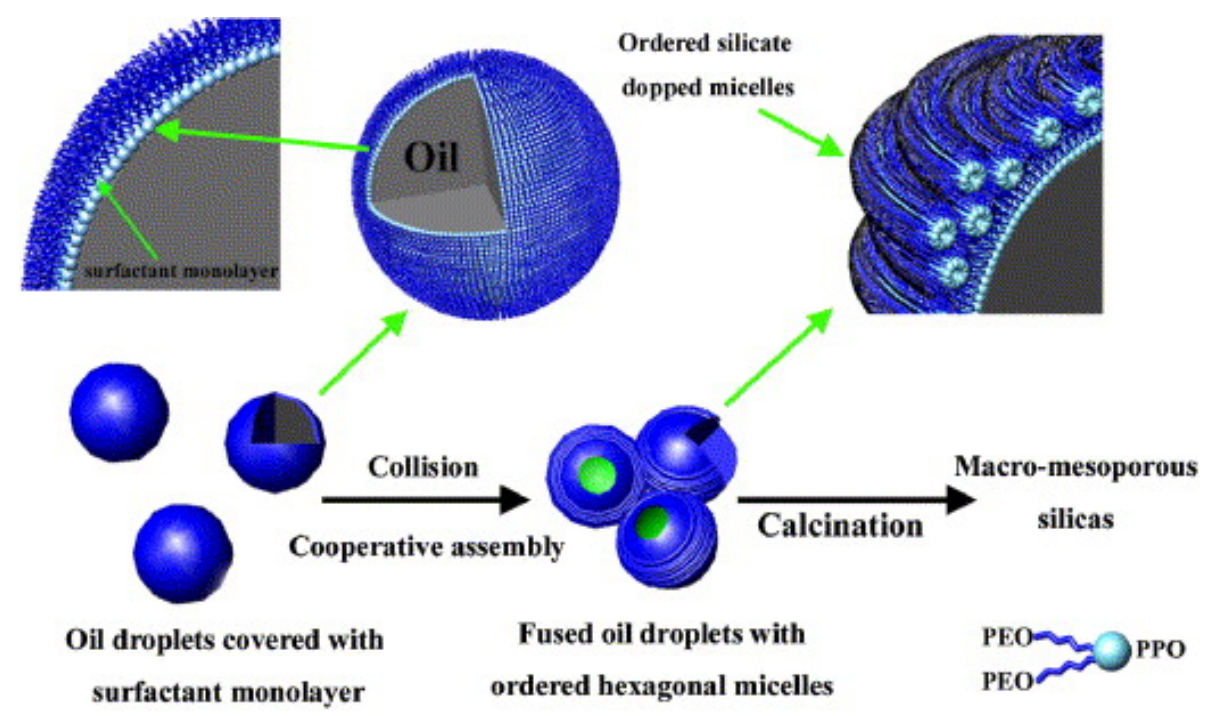

Scheme 1.Schematic representation of the formation of vesicular hierarchical structures.

It should also be mentioned that by employing different alkanes or different reaction compositions, the size of macropore is tunable. For example, macro-mesoporous silicas with 50$100 \mathrm{~nm}$ of macropore diameter have also been obtained using decane as cosolvents (Fig. S3), but totally different compositions and reaction temperatures were employed. This result confirms the significance of tuning the surfactant/silicate-dopped surfactant properties in the construction of the hierachical macro-mesoprous materials. In addition, to guarantee the ordered mesostructures of the macro-mesoporous silicas, a small amount of fluoride is of great importance in the emulsion system [16]. 
Mesoporous carbon materials with hierarchical porosity have wide applications in the fields of catalysis, adsorption, separation, etc [19]. Herein, the macro-mesoporous silicas are used as a template to construct hierarchically porous carbon materials by the nanocasting strategy. SEM images show that the obtained hierarchical porous carbon replica has similar morphology to that of the silica template (Fig. 3). HRSEM/TEM images (Fig. 3b-d) clearly demonstrated the multimodal pore system of these hierarchical macro-mesoporous carbon materials, which can be further confirmed by their nitrogen sorption isotherms (Fig. S4).
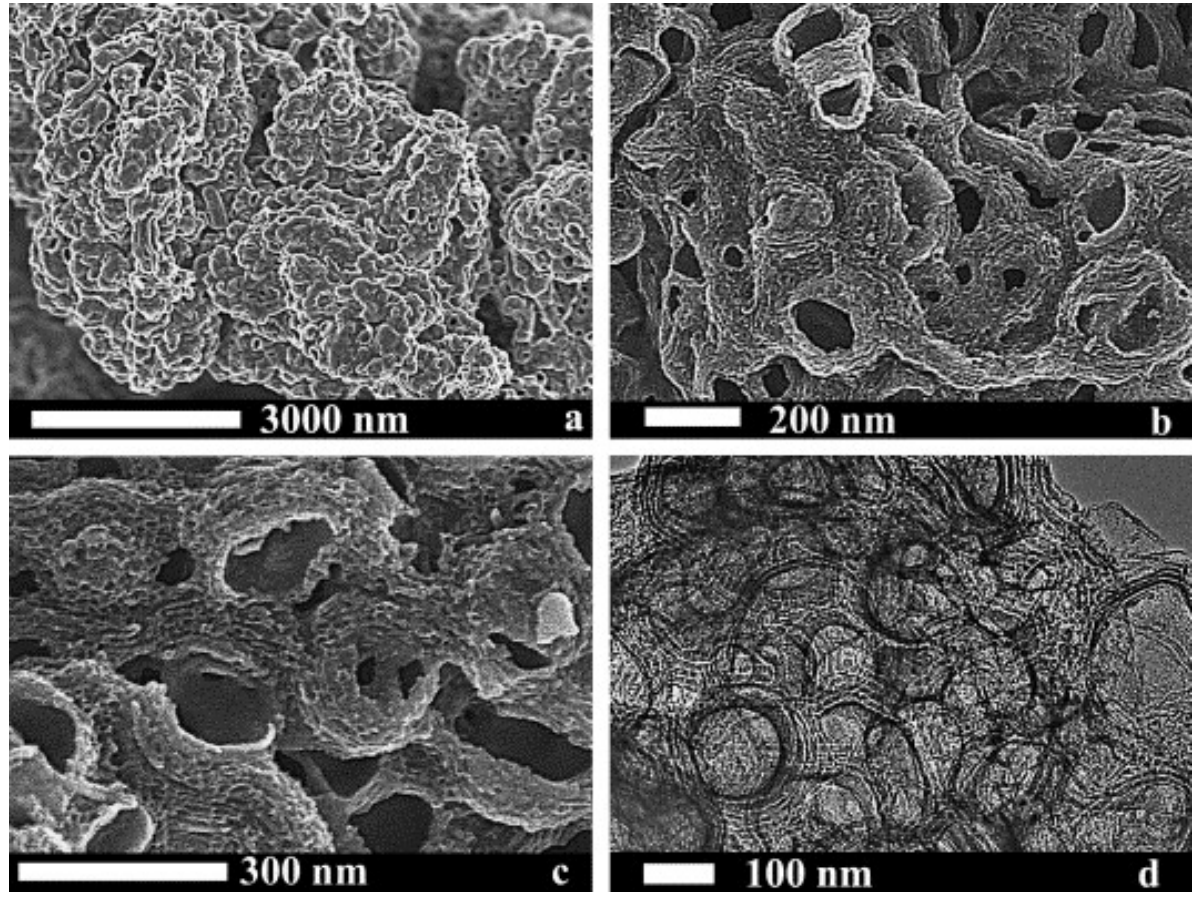

Fig. 3.SEM (a), HRSEM (b and c) and TEM (d) images of the macro-mesoporous carbon materials.

In summary, our results indicate that by balancing the hydrophobic-hydrophilic properties of the silicate-doped amphiphilic block copolymers system, miniemulsion droplets surrounded by the circular hexagonal-ordered silicate- surfactant complex were formed. After the condensation of silicate species, and the removal of surfactants and oil phase, novel hierarchical macromesoporous silicas are thus fabricated. Moreover, mesoporous carbon materials with hierarchical porosity are also successfully obtained by a nanocasting route. Further studies regarding continuously tuning the macropore size are in process.

\section{Acknowledgements}

We are grateful for the support of National Natural Science Foundation of China (No. 90206036) and the Ministry of Science and Technology of China (2005CB221405).

Appendix A. Supplementary data

Supplementary data associated with this article can be found, in the online version, at doi:10.1016/j.micromeso. 2006.11.016.

\section{References}

[1] T. Yanagisawa, T. Shimizu, K. Kuroda, C. Kato, Bull. Chem. Soc. Jpn. 63 (1990) 988.

[2] G.J.D. Soler-illia, C. Sanchez, B. Lebeau, J. Patarin, Chem. Rev. 102 (2002) 4093.

[3] H.P. Lin, C.Y. Mou, Science 273 (1996) 765; P. Yang, T. Deng, D. Zhao, P. Feng, D. Pine,

B.F. Chmelka, G.M. Whitesides, G.D. Stucky, Science 282 (1998) 2244; B.T. Holland, C.F. Blanford, A. Stein, Science 281 (1998) 538; M.W. Anderson, S.M. Holmes, N. Hanif, C.S. Cundy, Angew. Chem. Int. Ed. 39 (2000) 2707. 
[4] A. Stein, Adv. Mater. 15 (2003) 763, and references therein.

[5] Z.Y. Yuan, B.L. Su, J. Mater. Chem. 16 (2006) 663; H. Zhang, A.I. Cooper, Soft Matter. 1 (2005) 107.

[6] A. Imhof, J. Pine, Nature 389 (1997) 948.

[7] T. Sen, G.J.T. Tiddy, J.L. Casci, M.W. Anderson, Micropor. Mesopor. Mater. 78 (2005) 255; T. Sen, G.J.T. Tiddy, M.W. Anderson, Chem. Commun. (2003) 2182; F. Carn, A. Colin, M.F. Achard, H. Deleuze, E. Sellier, M. Birot, R. Backov, J. Mater. Chem. 14 (2004) 1370.

[8] D. Zhao, J. Feng, Q. Huo, N. Melosh, G.H. Fredrickson, B.F. Chmelka, G.D. Stucky, Science 279 (1998) 548.

[9] Q. Sun, P.J. Kooyman, J.G. Grossmann, P.H.H. Bomans, P.M. Frederik, P.C.M.M. Magusin, T.P.M. Beelen, R.A. van Santen, N.A.J.M. Sommerdijk, Adv. Mater. 15 (2003) 1097.

[10] W.W. Lukens, J.P. Schmidt-Winkel, D. Zhao, J. Feng, G.D. Stucky, Langmuir 15 (1999) 5403;

T. Amatani, K. Nakanishi, K. Hirao, T. Kodaira, Chem. Mater. 17 (2005) 2114; J. Shah, T.J. Pinnavaia, Chem. Mater. 17 (2005) 947.

[11] S. Schacht, Q. Huo, I.G. Voit-Martin, G.D. Stucky, F. Schüth, Science 273 (1996) 768.

[12] D.H.W. Hubert, M. Jung, P.M. Frederik, P.H.H. Bomans, J. Meuldijk, A.L. German, Adv. Mater. 12 (2000) 1286; B. Tan, H.J. Lehmler, S.M. Vyas, B.L. Knutson, S.E. Rankin, Adv. Mater. 17 (2005) 2368;

H. Hentze, S.R. Raghavan, C.A. McKelvey, E.W. Kaler, Langmuir 19 (2003) 1069; Y. Lu, H. Fan,

A. Stump, T.L. Ward, T. Rieker, C.J. Brinker, Nature 398 (1999) 223.

[13] P.T. Tanev, T.J. Pinnavaia, Science 271 (1996) 1267.

[14] J.L. Blin, A. Leonard, Z.Y. Yuan, L. Gigot, A. Vantomme, A.K. Cheetham, B.L. Su, Angew. Chem. Int. Ed. 42 (2003) 2872.

[15] W. Deng, M.W. Toepke, B.H. Shanks, Adv. Funct. Mater. 13 (2003) 61.

[16] H. Zhang, J.M. Sun, D. Ma, X.H. Bao, A. Klein-Hoffmann, G. Weinberg, D.S. Su, R. Schlögl, J. Am. Chem. Soc. 126 (2004) 7440; J.M. Sun, H. Zhang, D. Ma, Y. Chen, X.H. Bao,

A. Klein-Hoffmann, N. Pfänder, D.S. Su, Chem. Commun. (2005) 5343; J.M. Sun, D. Ma, H. Zhang, C. Wang, X.H. Bao, D.S. Su, A. Klein- Hoffmann, G. Weinberg, S. Mann, J. Mater. Chem. 16 (2006) 1507.

[17] J.S. Yu, S. Kang, S.B. Yoon, G. Chai, J. Am. Chem. Soc. 124 (2002) 9382.

[18] M. Antonietti, S. Fo“" rster, Adv. Mater. 15 (2003) 1323; G. Wanka, H. Hoffmann, W. Ulbricht, Macromolecules 27 (1994) 4145; P. Alexandridis, T. Alan Hatton, Colloids Surf. A. 96 (1995) 1.

[19] S.J. Han, K. Sohn, T. Hyeon Chem. Mater. 12 (2000) 3337; S.H. Joo, S.J. Choi, I. Oh, J. Kwak, Z. Liu, O. Terasaki, R. Ryoo Nature 412 (2001) 6843. 